\section{AMAMENTAÇÃO EM PREMATUROS: CARACTERIZAÇÃO DO BINÔMIO MÃE-FILHO E AUTOEFICÁCIA MATERNA}

\author{
Breastfeeding premature babies: characterization of the mother- \\ child binomial and maternal self-efficacy
}

\author{
Amamantamiento de prematuros: caracterización del binomio \\ madre-hijo y la autoeficacia materna
}

Artigo Original

\section{RESUMO}

Objetivo: Analisar a amamentação em prematuros relacionando as características do binômio mãe-filho e a autoeficácia materna. Métodos: Estudo do tipo transversal, descritivo, exploratório, com abordagem quantitativa, em uma maternidade pública do estado do Piauí, com 21 mães e seus filhos internados na Unidade de Cuidados Intermediários Neonatal. Aplicou-se um questionário sobre a caracterização materna, um check-list para avaliar a técnica da amamentação e a escala Breastfeeding Self-Efficacy Scale - Short Form. Resultados: 10 (47,6\%) eram adolescentes, 11 (52,4\%) solteiras, $10(47,6 \%)$ com baixo nível de escolaridade, $10(47,6 \%)$ com renda baixa, 12 (57\%) não possuíam vínculo empregatício, $15(71,4 \%)$ residiam no interior do estado, $13(61,9 \%)$ apresentaram partos cesarianos, $11(52,4 \%)$ eram multigestas, 12 eram (57,1\%) multíparas, 11 (52,4\%) tiveram experiência em amamentar, $12(57,1 \%)$ planejaram a gravidez, $16(72,5 \%)$ realizaram o prénatal com menos de seis consultas e não foram diagnosticadas doenças durante o pré-natal e $13(81,3 \%)$ não receberam orientações sobre o aleitamento materno. Quanto os recémnascidos, $18(85,7 \%)$ nasceram com peso inferior a $1500 \mathrm{~kg}, 10(47,6 \%)$ alimentavam-se com aleitamento materno misto/parcial, $16(76,2 \%)$ já haviam feito uso de sonda orogástrica para oferta do leite, $8(66,7 \%)$ tinham dificuldade da pega, 7 (58,3\%) sucção débil, 8 (66,7\%) deglutição desorganizada, $10(83,3 \%)$ vigília prejudicada, $9(75,5 \%)$ tinham pouca duração da mamada, $8(66,6 \%)$ com posicionamento desorganizado e $13(61,9 \%)$ das mães possuíam alta eficácia em amamentar. Conclusão: As mães do estudo mostraram-se com alta eficácia em amamentar seus filhos prematuros. Entretanto, na observação da técnica da mamada, esta se apresentou ineficaz.

Descritores: Amamentação; Prematuro; Mães; Autoeficácia; Enfermagem.

\section{ABSTRACT}

Objective: To analyse breastfeeding in premature babies, relating the characteristics of the mother-child binomial and maternal self-efficacy. Methods: Cross-sectional, descriptive, and exploratory study, with quantitative approach, in a public maternity hospital in the state of Piaui, Brazil, with 21 mothers and their children admitted to a Neonatal Intermediate Care Unit. A questionnaire on the maternal characterization, a checklist for assessment of the breastfeeding technique, and the Breastfeeding Self-Efficacy Scale - Short Form were applied. Results: Of the mothers, 10 (47.6\%) were teenagers, $11(52.4 \%)$ single, $10(47.6 \%)$ with low education level, 10 (47.6\%) with low income, 12 (57\%) had no employment bond, $15(71.4 \%)$ resided in the countryside of the state, 13 (61.9\%) had a caesarean section, 11 (52.4\%) had a previous pregnancy, 12 (57.1\%) were multiparous, 11 (52.4\%) had experience in breastfeeding, 12 (57.1\%) planned the pregnancy, 16 (72.5\%) had prenatal care with less than six visits, without any diagnosis of diseases, and 13 (81.3\%) did not receive guidance about breastfeeding. Regarding the newborns, 18 (85.7\%) weighed less than 1,500 kg at birth, 10 (47.6\%) were fed with mixed/partial maternal breastfeeding, 16 (76.2\%) had already used the orogastric feeding tube, 8 (66.7\%) had latch difficulties, 7 (58.3\%) poor suction, 8 (66.7\%) disorganized swallowing, 10 (83.3\%) reduced alertness, 9 (75.5\%) had

\author{
Antonia Mauryane Lopes ${ }^{(1)}$ \\ Grazielle Roberta Freitas da \\ Silva ${ }^{(1)}$ \\ Silvana Santiago da Rocha ${ }^{(1)}$ \\ Fernanda Valéria Silva Dantas \\ Avelino $^{(1)}$ \\ Lorena Sousa Soares $^{(2)}$
}

1) Universidade Federal do Piauí - UFPI Teresina (PI) - Brasil

2) Universidade Federal do Piauí - UFPI Floriano (PI) - Brasil
Recebido em: 22/05/2014 Revisado em: $11 / 07 / 2014$ Aceito em: 21/11/2014 
short length of feeding, 8 (66.6\%) with improper positioning, and 13 (61.9\%) had high efficacy in breastfeeding. Conclusion: The mothers showed high efficacy in breastfeeding their premature children. Considering the breastfeeding technique, however, this one was ineffective.

Descriptors: Breastfeeding; Premature; Mothers; Self Efficacy; Nursing.

\section{RESUMEN}

Objetivo: Analizar el amamantamiento de prematuros relacionando las características del binomio madre-hijo y la autoeficacia materna. Métodos: Estudio del tipo transversal, descriptivo, exploratorio de abordaje cuantitativo en una maternidad pública del estado de Piaui con 21 madres y sus hijos ingresados en la Unidad de Cuidados Intermediarios Neonatal. Se aplicó un cuestionario sobre la caracterización materna, un check-list para evaluar la técnica de amamantamiento y la escala Breastfeeding Self-Efficacy - Short Form. Resultados: 10 (47,6\%) eran adolescentes, $11(52,4 \%)$ solteras, $10(47,6 \%)$ con bajo nivel de escolaridad, 10 (47,6\%) con baja renta, 12 (57\%) no tenían vínculo laboral, 15 (71,4\%) vivían en los pueblos del estado, 13 (61,9\%) tuvieron cesáreas, 11 (52,4\%) eran multigestas, 12 eran (57,1\%) multiparas, 11 (52,4\%) tuvieron la experiencia de amamantar, 12 (57,1\%) habian planeado el embarazo, $16(72,5 \%)$ realizaron el prenatal con menos de seis consultas sin diagnóstico de enfermedades y 13 (81,3\%) no recibieron las orientaciones del amamantamiento materno. Respecto a los recién-nacidos, 18 (85,7\%) nacieron con el peso abajo de $1500 \mathrm{~kg}, 10$ (47,6\%) se alimentaban de la leche materna mixta/parcial, 16 (76,2\%) ya habian usado la sonda orogastrica para la oferta de la leche, 8 (66,7\%) tenian dificultad de pega al seno, 7 (58,3\%) tenían succión débil, 8 (66,7\%) deglución desorganizada, 10 (83,3\%) vigilia perjudicada, $9(75,5 \%)$ presentaron corta duración de la mamada, $8(66,6 \%)$ tenían la posición desorganizada y $13(61,9 \%)$ madres tenían elevada eficacia de amamantamiento. Conclusión: Las madres del estudio presentaron elevada eficacia de amamantar sus hijos prematuros. Sin embargo, en la observación de la técnica de la mamada, la misma no se presentó eficaz.

Descriptores: Lactancia Materna; Prematuro; Madres; Autoeficacia; Enfermería.

\section{INTRODUÇÃO}

A amamentação como prática social tem apresentado várias transformações ao longo do tempo devido aos benefícios a médio e longo prazo. Configura-se como uma ação estratégica no cenário da política pública, com influência na família, abrangência nos aspectos de ordem física, biológica, psicológica, cultural e social, além de possuir forte impacto no crescimento e desenvolvimento infantil.
O aleitamento materno exclusivo (AME) é uma forma de amamentação em que a criança recebe somente leite materno no seio ou ordenhado, sem presença de outros líquidos, salvo medicamentos, xaropes e vitaminas. É de extrema significância, pois atende a todas as necessidades nutricionais e imunológicas do recém-nascido ${ }^{(1)}$.

Diante dessas considerações, a recomendação sobre a oferta de leite materno é que seja realizada exclusivamente até os seis meses de vida e a manutenção da amamentação seja complementada até os dois anos de idade. Embora haja consenso de que o AME fornece inúmeros benefícios, sendo enaltecido e incentivado por programas que auxiliam a promoção, a prevalência ainda se encontra insatisfatória. Isso se constata nas principais capitais brasileiras ${ }^{(2)}$ e no Distrito Federal no ano de 2011, quando a prevalência do AME foi de $52,5 \%$ em crianças menores de três meses e $25,4 \%$ entre crianças de três a seis meses. Em outro estudo, mostrou-se que menor prevalência se encontra em bebês do sexo masculino, mães de 20 anos de idade sem escolaridade e que trabalham fora de casa ${ }^{(1)}$.

Um estudo retrospectivo e analítico que avaliou a prevalência do AME entre os recém-nascidos pré-termo de muito baixo peso evidenciou que a amamentação foi prevalente em $90 \%$ das crianças, das quais $50 \%$ foram amamentadas exclusivamente na alta hospitalar, entretanto, $51 \%$ foram desmamadas antes de seu sexto mês ${ }^{(3)}$. Deve-se levar em consideração que a amamentação neste público de prematuros torna-se desafiante, devendo ser incentivada e realizada em conjunto, com apoio do binômio mãe-filho. No estado do Piauí, um estudo com a finalidade de realizar um diagnóstico do aleitamento materno (AM) com 1.963 crianças menores de um ano em 45 municípios revelou que $80,23 \%$ encontravam-se em AM no dia da pesquisa. Verificou-se, ainda, que do total da amostra existia a probabilidade de $16 \%$ serem aleitadas por AME e 58\% para aleitamento materno aos 361 dias $^{(4)}$.

Esses dados, embora possam se apresentar animadores quando comparados com os dados mais antigos sobre o AME, ainda estão muito aquém do recomendado pela Organização Mundial de Saúde, pois ainda são frequentes os casos de insucesso e interrupção precoce do aleitamento. A amamentação, quanto mais cedo é iniciada e mantida, age como protetor contra a morbidade e a mortalidade infantil ${ }^{(5)}$.

O AM se caracteriza como alimento natural, de baixo custo e rico em nutrientes. Não obstante, as evidências já comprovaram que o leite materno, pelas peculiaridades na sua composição nutricional, é sem dúvida o alimento que garante um bom crescimento e desenvolvimento do recémnascido, em especial dos prematuros ${ }^{(1)}$.

Nesse sentido, a amamentação em prematuros ainda se comporta como um desafio, devido aos fatores que estão 
ligados à prematuridade, tais como: imaturidade do sistema neurológico, reflexo de sucção/deglutição prejudicada e dificuldade de manter em alerta por muito tempo. A presença desses mecanismos citados pode dificultar o início precoce da amamentação e, consequentemente, pode causar uma baixa incidência de êxito na amamentação de prematuros, especialmente em unidades neonatais de risco $^{(6)}$

A amamentação de prematuros, bem como a autoeficácia materna em amamentar são fatores que estão associadas e tornam-se essenciais para a detecção do problema e subsídio para possíveis formulações de estratégias que possam auxiliar a promoção e manutenção do AM, especialmente em prematuros. Nesse contexto, entende-se que este estudo se configura como pertinente e de grande importância, tendo como objetivo analisar a amamentação em prematuros, relacionando as características do binômio mãe-filho e a autoeficácia materna.

\section{MÉTODOS}

Estudo do tipo transversal, descritivo, exploratório, com abordagem quantitativa, realizado em uma maternidade pública do estado do Piauí, no período de julho a agosto de 2013.

Trata-se de uma maternidade pública, conveniada ao Sistema Único de Saúde (SUS), situada no Bairro Ilhotas, na Zona Sul do município de Teresina, capital do Piauí. A instituição é referência por atender a clientela local, municípios vizinhos e do interior do estado, certificada pelo Ministério da Saúde, em 2014, como referência nacional nos cuidados aos bebês prematuros por meio do Método Canguru. É considerada a maior maternidade do estado, responsável por $63 \%$ dos partos realizados, apresentando em médio 1.200 internações por mês ${ }^{(7)}$. Sua estrutura conta hoje com 278 leitos, dos quais 20 são destinados a uma unidade intensiva neonatal, 20 para uma semiunidade intensiva neonatal materno-infantil/unidade de médio risco e duas unidade de Método Canguru, com 17 leitos cada ${ }^{(7)}$. A missão da instituição é prestar assistência integral e de qualidade à mulher na idade fértil, desde a pré-concepção até a gestação de alto risco, seja em caráter de rotina ou de urgência/emergência.

A semiunidade intensiva neonatal (UCINCO) da referida instituição é um local de assistência aos cuidados intermediários e atende mensalmente uma média de 52 recém-nascidos, sendo $71 \%$ prematuros. No setor, há uma grande admissão de prematuros, porém com baixa rotatividade. $\mathrm{O}$ setor funciona 24 horas diárias, com equipe composta por médico, enfermeiro, técnicos de enfermagem, fonoaudiólogo, fisioterapeuta e nutricionista.
A escolha em pesquisar o tema e o cenário da pesquisa se deu mediante a compreensão da complexidade dos benefícios do aleitamento materno para o prematuros, por meio do contato e convívio com mães, bebês e profissionais atuantes, observados durante o estágio curricular, além de ser um campo rico para promoção de mudanças significativas com os resultados que serão encontrados na pesquisa.

A população do estudo foi composta por mães com filhos internados na UCINCO, sendo a amostra composta por 21 dessas mães, selecionadas de forma não probabilística por conveniência, a partir do seguinte critério de inclusão: mães que estavam amamentando e com filhos de prematuridade moderada (entre 31 e 34 semanas). Foram excluídas: mães que possuíssem impossibilidade mental que dificultasse o entendimento durante a aplicação dos instrumentos de coleta e mães de prematuros com má formação congênita que impossibilitasse a amamentação, como lábio leporino/ fenda palatina.

Os dados foram coletados por pesquisador treinado, que aplicou os seguintes instrumentos: 1) questionário para a caracterização materna relacionado a aspectos socioeconômicos, obstétricos e dos prematuros (situação civil, ocupação, nível de escolaridade, números de pessoas no domicílio, renda aproximada e procedência, número de gestações anteriores, tipo de parto atual e ocorrência ou não de aborto, bem como a caracterização dos prematuros quanto à idade, sexo, medidas antropométricas, Apgar ao nascer, alimentação atual e tratamento utilizado); 2) checklist de observação sistemática para avaliar a técnica de amamentação $^{(8)}$ e a escala Breastfeeding Self-Efficacy Scale - Short Form (BSES-F) para avaliar a autoeficácia materna mediante os escores encontrados.

Essa escala tem forma organizacional aleatória, constituída por 14 itens divididos em duas categorias de domínios: Técnico ( 8 itens - 1, 3, 4, 6, 11, 12, 13,14) e Pensamentos Intrapessoais (6 itens - 2, 5, 7, 8, 9,10). A primeira parte aborda aspectos do $\mathrm{AM}$, tais como: posição correta do bebê, conforto e outros. Já a segunda parte avalia desejo, motivação e satisfação com a experiência em amamentar. A eficácia materna distribui-se de acordo com as pontuações adquiridas nesta escala: eficácia baixa (14 a 32 pontos), eficácia média (33 a 51 pontos) e eficácia alta (52 a 70 pontos) ${ }^{(9)}$.

Iniciou-se a coleta de dados com a revisão dos prontuários, averiguando-se as mães aptas a participarem da pesquisa, as quais, individualmente, foram convidadas no momento em que se encontravam junto ao leito de seus respectivos filhos. Posteriormente, realizou-se a aplicação do instrumento referente às características maternas e dos prematuros. Alguns dados, tais como Apgar ao nascer, tipo de tratamento utilizado e tempo de internação, foram retirados dos respectivos prontuários, assim como os dados 
antropométricos, com ressalva para o peso atual, medido manualmente.

Em seguida, aplicou-se a escala BSES-SF e realizouse a observação sistemática não-participante das mães que estavam amamentando no momento da coleta, fazendo uso de um instrumento para avaliar a técnica de amamentação.

A análise dos dados incluiu a estatística descritiva simples das variáveis, com frequência relativa e média. A participação dos sujeitos se deu por meio da aceitação, mediante a assinatura de um Termo de Consentimento Livre e Esclarecido, de acordo com as diretrizes da Resolução no 466/2012 do Conselho Nacional de Saúde. O projeto foi aprovado pelo Comitê de Ética em Pesquisa da Universidade Federal do Piauí (UFPI), sob parecer $n^{\circ}$ 335.996.

\section{RESULTADOS}

Quanto às características sociodemográficas das 21 mães participantes, $10(47,6 \%)$ estavam com idade entre 15 a 20 anos, $11(52,4 \%)$ eram solteiras e $10(47,6 \%)$ possuíam nível de escolaridade entre 10 e 12 anos. A maioria das mães residia no interior do estado do Piauí, (15; 71,4\%), sendo que $14(66,7 \%)$ coabitam com cerca de 1 a 5 pessoas no mesmo domicílio. Quanto à renda familiar, $10(47,6 \%)$ revelaram viver um salário mínimo e 12 (57,1\%) não

Tabela I - Distribuição do perfil sociodemográfico das mães de prematuros internados na UCINCO (n=21). Teresina-PI, 2013

\begin{tabular}{|c|c|c|}
\hline Variáveis & $\mathbf{n}$ & $\%$ \\
\hline \multicolumn{3}{|l|}{ Idade (anos) } \\
\hline $15-20$ & 10 & 47,6 \\
\hline $21-26$ & 8 & 38,1 \\
\hline $27-32$ & 3 & 14,3 \\
\hline \multicolumn{3}{|l|}{ Situação Ocupacional } \\
\hline Estudante & 5 & 23,9 \\
\hline Desempregada sem carteira assinada & 2 & 9,5 \\
\hline Desempregada & 12 & 57,1 \\
\hline Dona do lar & 2 & 9,5 \\
\hline \multicolumn{3}{|l|}{ Situação Civil } \\
\hline Casada/União estável & 9 & 42,9 \\
\hline Solteira & 11 & 52,4 \\
\hline Separada & 1 & 4,7 \\
\hline \multicolumn{3}{|l|}{ Escolaridade (anos completos) } \\
\hline $1-5$ & 5 & 23,9 \\
\hline $6-9$ & 4 & 19,0 \\
\hline $10-12$ & 10 & 47,6 \\
\hline$>13$ & 2 & 9,5 \\
\hline \multicolumn{3}{|l|}{ Procedência } \\
\hline Teresina & 4 & 19,1 \\
\hline Interior do estado do Piauí & 15 & 71,4 \\
\hline Outro estado & 2 & 9,5 \\
\hline \multicolumn{3}{|c|}{ Números de pessoas que moram na casa } \\
\hline $1-5$ pessoas & 14 & 66,7 \\
\hline $6-11$ pessoas & 7 & 33,3 \\
\hline \multicolumn{3}{|l|}{ Renda familiar* } \\
\hline Sem nenhuma renda & 1 & 4,8 \\
\hline Somente Bolsa Família & 1 & 4,8 \\
\hline Um salário mínimo & 10 & 47.6 \\
\hline Inferior a um salário mínimo & 3 & 14,3 \\
\hline Superior a um salário mínimo & 6 & 28.5 \\
\hline \multicolumn{3}{|l|}{ Meio de informacão que tem em casa } \\
\hline Televisão & 15 & 71,4 \\
\hline Internet & 2 & 9,5 \\
\hline Rádio & 4 & 19,1 \\
\hline
\end{tabular}

*Valor do salário mínimo: R\$ 678,00 
possuíam vínculo empregatício. Sobre o principal meio de informação, 15 (71,4\%) afirmaram ser a televisão e entre as participantes, $14(66,7 \%)$ não faziam uso de fumo e 14 (66,7\%) não consumiam bebidas alcoólicas (Tabela I).

Quanto às características gineco-obstétricas, 11 $(52,4 \%)$ mães eram multíparas e tiveram parto vaginal. Ademais, 12 (57,1\%) apresentaram variação de paridade de um a dois partos. Vale destacar ainda que $11(52,4 \%)$, dentre as multíparas, revelaram que tiveram experiência em amamentar, com duração de 3 a 4 meses. Encontrou-se a maioria de partos cesarianos na gestação do filho atual (13; $61,9 \%)$, e $12(57,1 \%)$ das mães planejaram a concepção do filho (Tabela II).

Viu-se que $16(72,6 \%)$ das mães realizaram o prénatal mostrou-se satisfatório, entretanto, 7 (43,7\%) compareceram a menos de 6 consultas. Quanto às doenças gestacionais, $16(72,6 \%)$ não apresentaram esse diagnóstico. $\mathrm{O}$ estudo também evidenciou que da amostra estudada $13(81,3 \%)$ mães não receberam orientações sobre AM durante as consultas do pré-natal (Tabela II).

Tabela II - Caracterização gineco-obstétrico e gestação atual das mães de prematuros internados na UCINCO (n=21). Teresina-PI, 2013

\begin{tabular}{|c|c|c|}
\hline Variáveis & n & $\%$ \\
\hline \multicolumn{3}{|c|}{ HISTÓRICO GINECO-OBSTETRICO } \\
\hline Primigesta & 10 & 47,6 \\
\hline Multigesta & 11 & 52,4 \\
\hline \multicolumn{3}{|l|}{ Paridade } \\
\hline 1-2 partos & 12 & 57,1 \\
\hline 3-4 partos & 8 & 38,1 \\
\hline$>4$ partos & 1 & 4,8 \\
\hline Partos cesarianos & 11 & 52,4 \\
\hline Partos vaginais & 10 & 47,6 \\
\hline Números de prematuros & 1 & 4,7 \\
\hline Abortos & 1 & 4,7 \\
\hline \multicolumn{3}{|c|}{ Amamentou os outros filhos } \\
\hline Sim & 11 & 52,4 \\
\hline Não & 10 & 47,6 \\
\hline \multicolumn{3}{|c|}{ Por quanto tempo amamentou (meses) } \\
\hline $1-2$ & 4 & 19,1 \\
\hline $3-4$ & 10 & 47,6 \\
\hline $5-6$ & 7 & 33,3 \\
\hline \multicolumn{3}{|l|}{ GESTAÇÃO ATUAL } \\
\hline \multicolumn{3}{|l|}{ Tipo de parto } \\
\hline Parto normal & 8 & 38,1 \\
\hline Parto cesariano & 13 & 61,9 \\
\hline \multicolumn{3}{|l|}{ Gestação foi planejada } \\
\hline Sim & 12 & 57,1 \\
\hline Não & 9 & 42,9 \\
\hline \multicolumn{3}{|l|}{ Realizou pré-natal } \\
\hline Sim & 16 & 76,2 \\
\hline Não & 5 & 23,8 \\
\hline \multicolumn{3}{|c|}{ Números de consultas realizadas no pré-natal } \\
\hline$<6$ & 7 & 43,7 \\
\hline 6 & 3 & 18,7 \\
\hline$>6$ & 6 & 37,6 \\
\hline \multicolumn{3}{|c|}{ Doença diagnosticada durante o pré-natal } \\
\hline Sim & 5 & 23,8 \\
\hline Não & 16 & 76,2 \\
\hline \multicolumn{3}{|c|}{ Recebeu orientação no pré-natal sobre AM } \\
\hline Sim & 3 & 18,7 \\
\hline Não & 13 & 81,3 \\
\hline
\end{tabular}


Tabela III - Distribuição das características ao nascer dos prematuros internados na UCINCO (n=21). Teresina-PI, 2013.

\begin{tabular}{|c|c|c|}
\hline Variáveis & $\mathbf{n}$ & $\%$ \\
\hline \multicolumn{3}{|l|}{ Peso ao nascer (kg) } \\
\hline$>1500$ & 3 & 14,3 \\
\hline$<1500$ & 18 & 85,7 \\
\hline \multicolumn{3}{|l|}{ Sexo } \\
\hline Masculino & 13 & 61,9 \\
\hline Feminino & 8 & 38,1 \\
\hline \multicolumn{3}{|l|}{ Peso atual } \\
\hline$>2.500$ & 4 & 19,1 \\
\hline$<2.500$ & 17 & 80,9 \\
\hline \multicolumn{3}{|l|}{ Estatura } \\
\hline Meninos $<50 \mathrm{~cm}$ & 13 & 61,9 \\
\hline Meninas $>49 \mathrm{~cm}$ & 8 & 38,1 \\
\hline \multicolumn{3}{|l|}{ Perímetro cefálico $(\mathrm{cm})$} \\
\hline$=30$ & 5 & 23,8 \\
\hline$<30$ & 6 & 28,6 \\
\hline$>30$ & 7 & 33,3 \\
\hline Não consta & 3 & 14,3 \\
\hline \multicolumn{3}{|l|}{ Apgar no $1^{\circ}$ minuto de vida } \\
\hline$=7$ & 2 & 9,5 \\
\hline$<7$ & 6 & 28,6 \\
\hline$>7$ & 10 & 47,6 \\
\hline Não consta & 3 & 14,3 \\
\hline \multicolumn{3}{|l|}{ Apgar no $5^{\circ}$ minuto de vida } \\
\hline$=>7$ & 18 & 85,7 \\
\hline Não consta & 3 & 14,3 \\
\hline \multicolumn{3}{|l|}{ Alimentação atual do bebê } \\
\hline AME* & 8 & 38,1 \\
\hline Aleitamento materno misto/parcial & 10 & 47,6 \\
\hline $\mathrm{NP} *$ & 2 & 9,5 \\
\hline Somente leite artificial & 1 & 4,8 \\
\hline \multicolumn{3}{|l|}{ Fez uso de: } \\
\hline Sonda orogástrica & 16 & 76,2 \\
\hline Fototerapia & 2 & 9,5 \\
\hline Antibioticoterapia & 3 & 14,3 \\
\hline \multicolumn{3}{|l|}{ Tempo de internação (dias) } \\
\hline$<15$ & 14 & 66,7 \\
\hline$>15$ & 7 & 33,3 \\
\hline
\end{tabular}

*AME $=$ Aleitamento Materno Exclusivo.* NP = Nutrição parenteral

No que diz respeito aos prematuros, a idade gestacional ao nascer variou de 31 a 34 semanas; 18 (85,7\%) apresentaram peso inferior a $1500 \mathrm{~kg} ; 7(33,3 \%)$, perímetro cefálico maior que $30 \mathrm{~cm}$ e pequeno para idade gestacional (PIG). Houve predominância do sexo masculino $(13 ; 61,9 \%)$ e todos foram classificados com prematuridade moderada. Com relação ao Apgar no $1^{\circ}$ minuto, $10(47,6 \%)$ pontuaram maior que sete e $18(85,7 \%)$, mantiveram-se igual ou superior a sete no $5^{\circ}$ minuto de vida. Quanto à alimentação atual, $10(47,6 \%)$ alimentavam-se com aleitamento materno misto/parcial e $16(76,2 \%)$ já haviam feito uso de sonda orogástrica para oferta do leite (Tabela III).

Sobre a técnica da amamentação dos prematuros, apenas $12(57,1 \%)$ estavam amamentando seus filhos durante o período de coleta. Dessa forma, aproveitou-se o momento oportuno para observação sistemática nãoparticipante (Tabela IV).

Quanto à verificação da pega no mamilo, observou-se que $8(66,7 \%)$ não a realizavam corretamente e $6(50 \%)$ apresentavam dispneia frequente durante as sugadas. No 
que se refere à cor da pele, 12 (100\%) mantiveram-se normocoradas durante toda a mamada e 10 (83,3\%), não apresentaram regurgitação nasal-oral. Quanto ao estado de vigília, $10(83,3 \%)$ apresentaram sinais indicativos de problemas, como sonolência no início e durante a mamada, e $7(58,3 \%)$ apresentaram padrão de sucção débil. Quanto ao reflexo de busca, 10 (83,3\%) foram classificados como incompleto. No que se refere à duração da mamada, 9 (75,5\%) não apresentaram tempo suficiente e favorável ao AM, enquanto $8(66,7 \%)$ mostraram deglutição com presença de engasgo e tosse.
Quanto ao posicionamento na hora da mamada, 8 $(66,6 \%)$ dos bebês assumiram postura incorreta e $7(58,3 \%)$ das mães estavam com postura satisfatória.

Os resultados da avaliação da autoeficácia materna mostraram que $13(61,9 \%)$ das puérperas apresentaram escores variando de 55 a 61 pontos, indicando alta eficácia em amamentar prematuros. Além disso, analisando os itens da BSES-SF, verificou-se que no domínio técnico, bem como no domínio de pensamentos intrapessoais, as participantes apresentaram respostas "concordo" ou "concordo totalmente" para todos os itens, conforme apresentado na Tabela $\mathrm{V}$, reforçando a alta eficácia em amamentar prematuros.

Tabela IV - Distribuição dos parâmetros de avaliação da técnica de amamentação em prematuros internados na UCINCO $(\mathrm{n}=12)$. Teresina-PI, 2013.

\begin{tabular}{|c|c|c|}
\hline Variáveis & $\mathbf{n}$ & $\%$ \\
\hline \multicolumn{3}{|l|}{ Pega do mamilo } \\
\hline Correta & 8 & 66,7 \\
\hline Incorreta & 4 & 33,3 \\
\hline \multicolumn{3}{|l|}{ Frequência Respiratória } \\
\hline Eupneico & 4 & 33,4 \\
\hline Taquipneico & 1 & 8,3 \\
\hline Bradpneico & 2 & 8,3 \\
\hline Dispneia em toda a mamada & 6 & 50 \\
\hline \multicolumn{3}{|l|}{ Pele } \\
\hline Normocorada & 12 & 100 \\
\hline \multicolumn{3}{|l|}{ Regurgitação nasal-oral } \\
\hline Presente & 2 & 16,7 \\
\hline Ausente & 10 & 83,3 \\
\hline \multicolumn{3}{|l|}{ Vigília } \\
\hline Alerto & 2 & 16,7 \\
\hline Sonolento no inicio da mamada & 10 & 83,3 \\
\hline \multicolumn{3}{|l|}{ Sucção } \\
\hline Firme & 1 & 8,3 \\
\hline Débil & 7 & 58,3 \\
\hline Com pausas muitas longas & 4 & 33,3 \\
\hline \multicolumn{3}{|l|}{ Reflexo de busca } \\
\hline Presente & 2 & 16,7 \\
\hline Incompleto & 10 & 83,3 \\
\hline \multicolumn{3}{|l|}{ Duração da mamada (minutos) } \\
\hline 20 a 30 & 1 & 8,3 \\
\hline$<20$ & 9 & 75 \\
\hline$>20$ & 2 & 16,7 \\
\hline \multicolumn{3}{|l|}{ Deglutição } \\
\hline Ouve-se a deglutição & 4 & 33,3 \\
\hline Engasgo,tosse e outras alterações & 8 & 66,7 \\
\hline \multicolumn{3}{|l|}{ Postura do Bebê } \\
\hline Correta & 4 & 33,3 \\
\hline Incorreta & 8 & 66,7 \\
\hline \multicolumn{3}{|l|}{ Postura da mãe em amamentar } \\
\hline Correta & 7 & 58,3 \\
\hline Incorreta & 5 & 41,7 \\
\hline
\end{tabular}


Tabela V - Distribuição dos domínios técnicos e pensamentos intrapessoais da Breastfeeding Self-Efficacy Scale-Short Form (BSES - SF) encontrados nas mães de prematuros internados na UCINCO ( $\mathrm{n}=21)$. Teresina-PI, 2013.

\begin{tabular}{|c|c|c|c|c|c|c|c|c|c|c|}
\hline \multirow[t]{2}{*}{$\begin{array}{l}\text { Domínio Téenico } \\
(\mathrm{n}=21)\end{array}$} & \multicolumn{2}{|c|}{$\begin{array}{l}\text { Discordo } \\
\text { Totalmente }\end{array}$} & \multicolumn{2}{|c|}{ Discordo } & \multicolumn{2}{|c|}{$\begin{array}{c}\text { Ás vezes } \\
\text { Concordo }\end{array}$} & \multicolumn{2}{|c|}{ Concordo } & \multicolumn{2}{|c|}{$\begin{array}{l}\text { Concordo } \\
\text { Totalmente }\end{array}$} \\
\hline & $\mathrm{n}$ & $\%$ & $\mathbf{n}$ & $\%$ & $\mathbf{n}$ & $\%$ & $\mathrm{n}$ & $\%$ & $\mathbf{n}$ & $\%$ \\
\hline $\begin{array}{l}\text { 1. Eu sempre sinto quando o meu bebê } \\
\text { está mamando o suficiente }\end{array}$ & 4 & 19,0 & 4 & 19,1 & 3 & 14,3 & 6 & 28,6 & 4 & 19,0 \\
\hline $\begin{array}{l}\text { 3. Eu sempre alimento o meu bebê sem } \\
\text { usar leite em pó como suplemento }\end{array}$ & 3 & 14,3 & 4 & 19,0 & 4 & 19,1 & 4 & 19,0 & 6 & 28,6 \\
\hline $\begin{array}{l}\text { 4. Eu sempre percebo se meu bebê está } \\
\text { pegando o peito direitinho durante toda } \\
\text { a mamada }\end{array}$ & 1 & 4,8 & 2 & 9,5 & 6 & 28,6 & 8 & 38,0 & 4 & 19,1 \\
\hline $\begin{array}{l}\text { 6. Eu sempre posso amamentar mesmo } \\
\text { se o meu bebê estiver chorando }\end{array}$ & & -- & 8 & 38,1 & 2 & 9,5 & 8 & 38,0 & 3 & 14,4 \\
\hline $\begin{array}{l}\text { 11. Eu sempre amamento meu bebê em } \\
\text { um peito e depois mudo para o outro }\end{array}$ & & -- & 4 & 19,0 & 6 & 28,6 & 6 & 28,6 & 5 & 23,8 \\
\hline $\begin{array}{l}\text { 12. Eu sempre continuo amamentando } \\
\text { meu bebê a cada alimentação dele }\end{array}$ & 3 & 14,2 & 3 & 14,2 & 5 & 23,8 & 5 & 23,8 & 4 & 19,0 \\
\hline $\begin{array}{l}\text { 13. Eu sempre consigo adequar as } \\
\text { minhas necessidades às necessidades } \\
\text { do bebê. }\end{array}$ & & & 5 & 23,8 & 4 & 19,0 & 5 & 23,8 & 7 & 33,3 \\
\hline $\begin{array}{l}\text { 14. Eu sempre sei quando meu bebê } \\
\text { terminou a mamada }\end{array}$ & 1 & 4,8 & 8 & 38,0 & 1 & 4,8 & 5 & 23,8 & 6 & 28,6 \\
\hline \multirow[t]{2}{*}{$\begin{array}{l}\text { Domínios Intrapessoais } \\
(\mathrm{n}=21)\end{array}$} & \multicolumn{2}{|c|}{$\begin{array}{l}\text { Discordo } \\
\text { Totalmente }\end{array}$} & \multicolumn{2}{|c|}{ Discordo } & \multicolumn{2}{|c|}{$\begin{array}{l}\text { Ás vezes } \\
\text { Concordo }\end{array}$} & \multicolumn{2}{|c|}{ Concordo } & \multicolumn{2}{|c|}{$\begin{array}{c}\text { Concordo } \\
\text { Totalmente }\end{array}$} \\
\hline & $\mathbf{n}$ & $\%$ & $\mathrm{n}$ & $\%$ & $\mathrm{n}$ & $\%$ & $\mathbf{n}$ & $\%$ & $\mathrm{n}$ & $\%$ \\
\hline $\begin{array}{l}\text { 2. Eu sempre lido com a amamentação } \\
\text { com sucesso, da mesma forma que eu } \\
\text { lido com outros desafios }\end{array}$ & 1 & 4,8 & 4 & 19,1 & 2 & 9,5 & 12 & 57,1 & 2 & 9,5 \\
\hline $\begin{array}{l}\text { 5. Eu sempre lido com a amamentação } \\
\text { de forma a me satisfazer. }\end{array}$ & 2 & 9,5 & & -- & 6 & 28,6 & 6 & 28,6 & 7 & 33.3 \\
\hline $\begin{array}{l}\text { 7. Eu sempre sinto vontade de continuar } \\
\text { amamentando }\end{array}$ & & -- & 1 & 4,8 & 2 & 9,5 & 7 & 33,3 & 11 & 52,4 \\
\hline $\begin{array}{l}\text { 8. Eu sempre posso dar de mamar } \\
\text { confortavelmente na frente de pessoas } \\
\text { da minha família }\end{array}$ & 1 & 4,8 & 3 & 14,2 & 4 & 19,0 & 4 & 19,1 & 9 & 42,9 \\
\hline $\begin{array}{l}\text { 9. Eu sempre fico satisfeita com a } \\
\text { minha experiência de amamenta }\end{array}$ & & -- & 2 & 9,5 & 7 & 33,3 & 3 & 14,3 & 9 & 42,9 \\
\hline $\begin{array}{l}\text { 10. Eu sempre posso lidar com o fato } \\
\text { de que amamentar exige tempo }\end{array}$ & 2 & 9,5 & 2 & 9,5 & 4 & 19,1 & 9 & 42,8 & 4 & 19,1 \\
\hline
\end{tabular}




\section{DISCUSSÃO}

O presente estudo apresenta mães em sua maioria adolescentes, solteiras, com baixo nível de escolaridade e baixa renda, perfil que indica uma faixa etária associada à menor duração do AM, um nível de instrução e de estado civil que favorecem a decisão tardia da forma de alimentar o bebê e, consequentemente, comportam-se como fortes aliados ao desmame precoce ${ }^{(6,10,11)}$. Entretanto, apresentam alta eficácia no aleitamento materno.

É sabido que as mães de baixa renda são particularmente vulneráveis a um resultado insatisfatório na amamentação e possuem baixa eficácia ${ }^{(12)}$. Porém, quanto maior for a renda familiar, melhor o nível de autoeficácia na amamentação, sugerindo que quanto menor a renda familiar, mais precoce pode ser a interrupção do $\mathrm{AME}^{(13)}$. Entretanto, no presente estudo, o nível de autoeficácia foi identificado como alto, apesar da baixa renda familiar das participantes, o que pode sugerir orientações adequadas no período de internação do bebê pré-termo. Porém, por não ser um estudo longitudinal, não foi possível conhecer a continuidade do aleitamento materno exclusivo.

Diante desse enfoque, acredita-se que o sucesso da amamentação depende de medidas favoráveis que possam influenciar os envolvidos de forma satisfatória, sempre levando em consideração os fatores que estão diretamente relacionados às mães e aos situacionais das condições de vida.

Sobre o consumo de álcool, tabagismo e uso de drogas, aproximadamente mais da metade das mães não faziam uso de nenhuma das modalidades de drogas lícitas ou ilícitas. O lactente filho de mãe que usa álcool, apesar de sugar de forma mais vigorosa, ingere menos leite por tempo de sucção, fato não percebido pela mãe, o que dificulta a amamentação $^{(14)}$.

Vale destacar que a presença de multigestação, multiparidade, com uma variação de um a dois partos, pouca ocorrência de aborto e experiência em amamentar filhos anteriores com duração de 3 a 4 meses encontrados em aproximadamente mais da metade das mães pesquisadas são vistos como fatores que favorecem a autoeficácia da mãe em amamentar e melhora consideravelmente os índices da oferta do $\mathrm{AM}^{(15)}$.

É interessante ressaltar que algumas atitudes, em especial a experiência de amamentar, podem interferir sobre a promoção do AM, principalmente se as experiências anteriores forem negativas ${ }^{(9)}$. Neste estudo, as experiências anteriores de amamentação não foram investigadas, mas o conhecimento sobre esse aspecto é importante para o direcionamento do aconselhamento do profissional de saúde. Nesse sentido, investigar se a amamentação foi bem sucedida ou se aconteceram complicações pode direcionar a abordagem correta do profissional com a mãe, com um aconselhamento sistemático, diretivo e integral.

Com relação ao número de cesarianas, houve predominância nas gestações atuais, pois grande parte da amostra realizou esse tipo de parto. Um levantamento realizado nas capitais brasileiras aponta que as taxas de cesáreas revelaram um salto de $38 \%$ de todos os partos em 2000 para 54\% em 2011, e são vários os motivos que levam a esse aumento, porém, alguns ainda são desconhecidos ${ }^{(5,16)}$.

Acredita-se que esse percentual esteja relacionado ao perfil de risco das gestantes e à magnitude da prematuridade. A realização do pré-natal teve grande êxito, embora o número de consultas apareça abaixo do proposto pelo Ministério da Saúde. Mães que realizaram menos de seis consultas apresentaram duração mediana do AM menor do que as que realizaram seis ou mais consultas $^{(17)}$. Para reforçar, estudos constatam baixa eficácia em amamentação nas mães que não realizaram todas as consultas pré-natais ${ }^{(8,9)}$.

Um dado preocupante foi o fato de mais da metade das mães revelarem não ter recebido informações sobre AM durante o pré-natal. Esse fator prejudica substancialmente a adesão à sua prática, visto que o pré-natal é uma assistência prestada à saúde materna e neonatal, bem como é um momento propício para o casal receber as orientações necessárias e sentir-se motivado e receptivo às informações, sendo uma medida protetora para maior duração da $\operatorname{amamentação}^{(17)}$.

Diante dessa informação, cabe ressaltar que essa realidade pode estar atrelada à fragilidade da assistência, à inconsistência da equipe de saúde, a pouca interação ou falta de adequação de conhecimento prévio da exata forma de aconselhar, bem como pode ser um alerta para a reestruturação na qualidade das consultas de pré-natal.

Um dado positivo revelado por este estudo foi o planejamento da gravidez. Aproximadamente metade da amostra planejou a concepção do filho e é sabido que mães que planejaram o filho têm melhor enfrentamento para amamentar $^{(18,19)}$. Esse aspecto torna-se muito significante e animador, pois traduz ações de comprometimento, organização e melhoram as altas taxas de desmame precoce. No entanto, estranharam-se esses resultados, que são distintos de muitos estudos anteriores nessa clientela. Acredita-se que a timidez na hora das respostas possa ter inibido as mães, fato que propiciou a mistificação de certas informações.

A idade gestacional, que variou de 31 a 34 semanas, assim como o peso ao nascer, inferior a $1.500 \mathrm{~g}$, e PIG constituem resultados preocupantes para a eficácia do AM. O prematuro de baixo peso, ao nascer, apresenta características singulares que requerem uma adaptação complexa ao meio 
extrauterino, aspectos biológicos e sociais ${ }^{(20)}$. No entanto, distúrbios da prematuridade e tamanho ao nascer não apresentam um efeito sobre a duração do $\mathrm{AM}^{(21)}$.

Nesta pesquisa, os prematuros apresentaram-se vigorosos tanto no primeiro como no quinto minuto de vida. Essa vantagem caracteriza-os a priori como sadios, com boa vitalidade e disposição para o incentivo ao AM. O número de prematuros que estavam sendo alimentados com leite materno humano em AM misto/parcial superou consideravelmente o número do AME, sendo que $47 \%$ das mães revelaram que seus filhos recebem suplementação láctica artificial do banco de leite da maternidade. Esses resultados são também encontrados nas Unidades de Terapia Intensivas Neonatais brasileiras ${ }^{(21)}$. O banco de leite humano do cenário do estudo sempre apresenta estoque de oferta de leite humano pasteurizado muito abaixo da necessidade de suporte para assistência de mais de 80 recém-nascidos pré-termos, os quais estão internados nas suas dependências ${ }^{(22)}$. Infelizmente, embora a instituição incentive, apóie e tenha profissionais atuantes na busca ativa por doadoras de leite, dentro do próprio hospital e externamente, ainda há desconhecimento ou falta de conscientização por parte das mães, para efetivação eficaz do aporte de leite aos prematuros.

A baixa incidência do AME nesses ambientes de unidades neonatais pode estar relacionada ao baixo peso ao nascer e à idade gestacional. Esses fatores determinam um tempo maior de internação e consequentemente prejudicam a amamentação $^{(23)}$. Neste estudo, a maior parte dos prematuros usou sonda orogástrica enquanto internados. Fato esperado para essa clientela ${ }^{(24)}$, haja vista que a maioria é de prematuros, que não conseguem obter sincronia da respiração/deglutição, dificultando inicialmente a sucção, sendo necessária a utilização da sonda orogástrica para alimentação.

Os prematuros estavam com menos de 15 dias de internação durante o período de coleta, porém, sabe-se que o tempo de permanência nessas unidades está relacionado com diagnóstico clínico neonatal e recuperação. No entanto, quando esse período passa a ser mais prolongado, torna-se uma barreira para a formação de vínculo entre mãe e filho e para o AM. Embora se deva considerar que o início da dieta depende da evolução clínica dos prematuros, é recomendável que eles recebam leite materno o mais precocemente possível e que tal prática seja considerada ${ }^{(25)}$.

Dessa forma, essa clientela investigada carece de uma ação mais direcionada, humanizada e preventiva, visando uma forte contribuição, com início precoce e, consequentemente, uma influência positiva na manutenção. Acredita-se que a internação de um filho dentro das unidades neonatais é desafiadora e as mães devem receber todo o conforto para que possam participar eficazmente nesse processo e minimizar as complicações.

Associado ao fato de a internação do filho ser desafiadora, a maior parte dos prematuros avaliados ainda apresentou sinais comportamentais desfavoráveis para a prática do $\mathrm{AM}$, tais como: pega incorreta no mamilo, frequência respiratória com presença de dispneia em toda a mamada, padrão sonolento no início da mamada e sucção débil. Resultados semelhantes foram encontrados em estudo realizado no Oeste do Paraná, no município de Cascavel, que avaliou o AM em prematuros no primeiro mês e pós-alta, também encontrando sinais desfavoráveis à amamentação ${ }^{(25)}$.

Para que a amamentação aconteça de forma adequada é necessário que os bebês possuam coordenação rítmica de sucção, deglutição e respiração, mas, devido à prematuridade, eles não apresentam sucção firme e vigorosa, com sugadas rápidas ao ritmo de $2: 1$, e quando apresentam não são capazes inicialmente de coordenar essa $\operatorname{sincronia}^{(26)}$.

Nesta pesquisa, o reflexo de busca esteve na maioria das vezes incompleto e aproximadamente mais da metade apresentou engasgo, tosse durante a mamada, conseguindo realizar poucos minutos de mamada. Esses dados se assemelham a outros estudos ${ }^{(25)}$. A presença do reflexo de busca se comporta com o precursor para a pega correta do mamilo, direcionando os lábios e a língua para abocanhar ${ }^{(27)}$.

Encontrou-se ainda ausência de posicionamento coordenado entre binômio mãe-filho, uma vez que a posição da mãe durante a mamada foi classificada como boa, mas, entre os prematuros, mais da metade assumiu posição errada durante a observação.

Esses parâmetros sugerem prática do AM negativa, em especial na manutenção da lactação, embora tais limitações não constituam um obstáculo não superável. Diante dessa realidade, é esperado e aceitável que os profissionais não forneçam somente as vantagens do AM nessa clientela, mas que também lhe ensinem a técnica correta e acompanhem esse momento mais de perto. Dessa forma, o profissional estará ao mesmo tempo fornecendo apoio social para que as mães se sintam confiantes e diminuindo a incidência do desmame precoce, ainda dentro da maternidade.

O grau de autoeficácia em amamentação presente nas mães foi alto, o que parece estar relacionado ao fato de as mães mostrarem-se capazes de reconhecer o manejo técnico da sucção do bebê, bem como ao fato de acreditarem que uma forma de acalmá-lo seria a oferta do seio na hora do choro.

Dados semelhantes em estudos que utilizaram a BSES-F comprovaram que as mães com alta eficácia são significantemente mais propensas a amamentarem e fazê-la 
exclusivamente desde a primeira semana até quatro meses após o parto do que as mães com baixa eficácia ${ }^{(28) .}$

É fundamental salientar que a prematuridade dos bebês sugere condições da sucção débil apresentadas após o nascimento, as quais, associadas ao pouco conhecimento das mães sobre sinais de comportamentos do bebê durante a amamentação, podem contribuir para pior eficácia ao $\operatorname{amamentar}^{(25)}$.

A percepção das mães sobre se o bebê mamou suficiente ou na decisão de não oferecer AME podem indicar baixa eficácia e reforçam que a amamentação não é uma habilidade total da mãe, mas algo que precisa ser aprendido por elas e, nesses casos, acompanhar cada binômio é uma necessidade nessas unidades de cuidados neonatais.

A elevada autoeficácia em amamentar está relacionada com o início e a exclusividade do $\mathrm{AM}^{(29)}$ e não pode ser dissociada da motivação pessoal da puérpera. Além disso, as mães, quando prontas para o desafio da amamentação, como mostrado pelas participantes do presente estudo, mostramse confiantes e com vontade de amamentar, principalmente em condições especiais como a dessas mulheres que têm seu filho, recém-nascido, internado em UTI.

\section{CONCLUSÃO}

As mães mostraram-se com alta eficácia em amamentar seus filhos prematuros, reafirmando enfrentar a amamentação da mesma forma que superam os desafios cotidianos. Entretanto, na observação da técnica da mamada, esta se apresentou ineficaz. As evidências até aqui elencadas agem positivamente como impacto favorável para o aumento dos índices do AM, em especial dentro das UCINCO, assim como no ambiente domiciliar.

\section{AGRADECIMENTOS}

Agradeço à maternidade púbica de Teresina pela autorização para a realização do trabalho.

\section{REFERÊNCIAS}

1. Ministério da Saúde (BR), Secretaria de Atenção à Saúde, Departamento de Ações Programáticas e Estratégicas. Cuidados gerais: Atenção à saúde do recém-nascido: guia para os profissionais de saúde. Brasília: Ministério da Saúde; 2011.

2. Saldiva SRDM, Venâncio SI, Gouvéia AGC, Castro ALS, Escuder MML, Giugliani ERJ. Influência regional no consumo precoce de alimentos diferentes do leite materno em menores de seis meses residentes nas capitais brasileiras e Distrito Federal. Cad Saúde Pública. 2011; 27(11):2253-62.
3. Sassá AH, Lopes CG, Bengozi TM, Rossetto EG, Sousa SNDH, Dalmas JC. Prevalence of breastfeeding in preterm infants with very low birth weight in the first six months of life. Acta Sci Health Sci. 2013;35(2):151-9.

4. Ramos CV, Almeida JAG, Alberto NSMC, Teles JBM, Saldiva SRDM. Diagnóstico da situação do aleitamento materno no Estado do Piauí, Brasil. Cad Saúde Pública. 2008:24(8): 1753-62.

5. World Health Organization - WHO. Infant and young child feeding: model chapter for textbooks for medical students and allied health professionals. 2009. [acesso em 2013 Ago 16]. Disponível em http://www.who.int/ nutrition/publications/infantfeeding/9789241597494/ en/.

6. Santos SAT, Dittz SE, Costa RP. Práticas favorecedoras do aleitamento materno ao recém- nascido prematuros. Rev Enferm Cent-Oeste Min. 2012;2(3):438-50.

7. Secretária de Saúde do Estado do Piauí (BR). Ministério da Saúde certifica Método canguru da Maternidade Evangelina Rosa. Teresina; 2015 [acesso em 2014 Nov 02]. Disponível em: http://www.saude.pi.gov. br/noticias/2014-08-29/6004/ministerio-da-saudecertifica-metodo-canguru-da-maternidade-evangelinarosa.html.

8. Tavares LAM. Proposta de formulário de observação da amamentação para RN pré-termo adaptado no modelo da UNICEF (1993/2004) [acesso em 2013 Ago 13]. Disponível em: http://www.aleitamento.com/ amamentacao/categoria.asp?cod_conteudo_sub $=51$.

9. Dodt RCM. Aplicação e validação da Breastfeeding Self-Efficacy Scale: Short Form (BSES-SF) em Puérperas [dissertação]. Fortaleza: Universidade Federal do Ceará; 2008 [acesso em 2013 Set 09]. Disponível em: http://www.repositorio.ufc.br:8080/ri/ handle/123456789/2018.

10. Carrascoza KC, Costa Júnior AL, Moraes ABA. Fatores que influenciam o desmame precoce e a extensão do aleitamento materno. Estud Psicol. 2005;22(4):433-40.

11. Escobar AMU, Ogawa AR, Hiratsuka M, Kawashita MY, Teruya PY, Grisis S et al. Aleitamento materno e condições socioeconômico culturais: fatores que levam ao desmame precoce. Rev Bras Saúde Matern Infant. 2002;2(3):253-61.

12. Gregory A, Penrose K, Morrison C, Dennis CL, MacArthur C. Psychometric properties of the Breastfeeding Self-E/cacy Scale-Short Form in an ethnically diverse U.K. sample. Public Health Nurs. 2008;25(3):278-84. 
13. Tavares MC, Aires JS, Dodt RCM, Joventino ES, Oriá MOB, Ximenes LB. Application of Breastfeeding SelfEfficacy Scale to post-partum women in rooming-in care: a descriptive study. Online Braz J Nurs (Online). 2010;9(1):87-91

14. Mennella JA, Pepino Y, Teff KL. Acute alcohol consumption disrupts the hormonal milieu of lactating women. J Clin Endocrinol Metab. 2005;90(4):1979-85.

15. Uchoa JL. Autoeficácia das mulheres no ciclo gravídicopuerperal em amamentar [dissertação]. Fortaleza: Universidade Federal do Ceará; 2012 [acesso em 2013 Set 01]. Disponível em: http://www.repositorio.ufc. br:8080/ri/handle/123456789/4133

16. Wutke $\mathrm{K}$, Dennis CL. The reliability and validity of the Polish version of the Breastfeeding Self-Efficacy Scale-Short Form. Int J Nurs Stud. 2007;44(8):143946.

17. Oliveira MGOA, Lira PIC, Batista Filho M, Lima MC. Fatores associados ao aleitamento materno em dois municípios com baixo desenvolvimento no Nordeste brasileiro. Rev Bras Epidemiol. 2013;16(1):178-89.

18. Dodt RCM. Elaboração e validação de tecnologia educativa para autoeficácia da amamentação [tese]. Fortaleza: Universidade Federal do Ceará; 2011 [acesso em 2013 Set 01]. Disponível em: http://www. repositorio.ufc.br:8080/ri/handle/123456789/4127

19. Haughton J, Gregorio D, Pérez-Escamilla R. Factors Associated With Breastfeeding Duration Among Connecticut Special Supplemental Nutrition Program for Women, Infants, and Children (WIC) Participants. J Hum Lact. 2010;26(3):266-73

20. Vieira CS, Melo DF. O seguimento da saúde da criança pré-termo de baixo peso egresso da Unidade de Terapia Neonatal. Texto \& Contexto Enferm. 2009;18(1):74-82

21. Sucena LP, Furlan MFFM. A incidência da utilização de leite materno ordenhado em uma unidade de terapia intensiva neonatal e caracterização do recém-nascido. Arq Ciênc Saúde. 2008; 15(2):82-9.
22. Secretária de Saúde do Estado do Piauí (BR). Banco de Leite da Maternidade Evangelina Rosa está em estoque baixo. Teresina; 2015 [acesso em 2014 Nov 01]. Disponível em: http://www.saude. pi.gov.br/noticias/2015-04-13/6282/banco-de-leitematernidade-evangelina-rosa-esta-com-estoque-baixo. html

23. Martins EL, Padoin SMM, Rodrigues AP, Zuge SS, Paula CC, Trajanh CT, et al. Caracterização de recémnascido de baixo peso internado em uma unidade de terapia intensiva neonatal. Rev Enferm UFSM. 2013:3(1):155-63.

24. Boucher CA. Breastfeeding experiences in the NICU. Neonatal Netw. 2011;30(1):21-8 .

25. Gubert JK, Vieira CS, Oliveira BRG, Delatore S, Sanches MM. Avaliação do aleitamento materno de recém-nascidos prematuros no primeiro mês pós alta. Ciênc Cuid Saúde 2012;11(1):146-55.

26. Yamamoto RCC, Keske-Soares M, Weinmann ARM. Características da sucção nutritiva na liberação da via oral em recém-nascidos pré-termo de diferentes idades gestacionais. Rev Soc Bras Fonoaudiol. 2009;14(1): 98-105.

27. Sanches MT. Manejo clínico das disfunções orais na amamentação. J Pediatr. 2004;80(5 Supl):S155-S162.

28. Blyth R, Creedy DK, Dennis CL, Moyle W, Prantt $\mathrm{J}$, Vries SM. Effect of maternal confidence on breastfeeding duration: an Application of breastfeeding self-efficacy theory. Birth. 2002;29(4):278-84.

29. Oriá MO, Ximenes LB. Tradução e adaptação cultural da Breastfeeding Self-Efficacy Scale para o português. Acta Paul Enferm. 2010;23(2):230-8.

\section{Endereço para correspondência:}

Antonia Mauryane Lopes

Campus Universitário Ministro Petrônio Portella

Bairro: Ininga

CEP: 64049-550 - Teresina - PI - Brasil

Email: lopes_mauryane_lopes@hotmail.com 